\title{
Recurrence of orbital cysts in the branchio-oculo-facial syndrome
}

\author{
D W Fielding, A E Fryer
}

\begin{abstract}
Two sibs with the branchio-oculo-facial syndrome are reported. They both have orbital haemangiomatous cysts, which is a previously unreported feature. Both parents are clinically normal and unrelated. This disorder has been reported showing autosomal dominant transmission so this family could represent either an autosomal recessive form or germline mosaicism for the dominant gene.
\end{abstract}

The branchio-oculo-facial syndrome (haemangiomatous branchial cleft syndrome, pseudocleft of the upper lip, cleft lip palate, and haemangiomatous branchial cleft syndrome) was described by Hall et al in 1984. The features include haemangiomatous branchial cysts, ocular anomalies (microphthalmos, anophthalmos, myopia, cataract, colobomas), cleft lip and palate (can be a pseudocleft produced by thick, fibrous, vertical ridges in the philtrum), and unusual facies (hypertelorism, upward slanting palpebral fissures, broad nose, and posteriorly rotated ears with thin helix, prominent antihelix, and upturned lobules). Autosomal dominant inheritance has been reported. We report a recurrence in a sibship with normal parents and where the ocular manifestations of the disorder in the sibs are large haemangiomatous orbital cysts.

\section{Case reports}

The first child of unrelated parents was a male. Pregnancy was complicated by an appendicectomy operation at three to four weeks, which was further complicated by an oculogyric crisis as a reaction to prochlorperazine. Birth was uncomplicated, but immediately after birth a cystic swelling appeared in the right orbit which increased in size dramatically. The left orbit appeared small. The eyeballs could not be located on neonatal examination but eyelids and eyelashes were present. Haemangiomatous birthmarks were noted over each side of the neck (fig 1). He also had anomalous retroverted ears and an "upper lip that looks as if it had just escaped a cleft". His unusual facial appearance is shown in fig 2. Chromosomes were normal.

An ultrasound scan of the orbits showed fluid filled cystic lesions. A CT scan showed that both globes were present but that they had been compressed upwards into the roof of the orbit by the bilateral masses. The lower lid cystic lesions appeared to be haemangiomas. A diagnosis of the branchio-oculo-facial syndrome was made.
He underwent surgery for removal of the bilateral orbital cysts at the age of 2 years 9 months. The cysts were found to be attached to the globe inferiorly and there was virtually no globe in the left eye and only a small remnant globe in the right. He was fitted with artificial eyes. The haemangiomatous lesions on both sides of the neck were excised at the age of $4 \frac{1}{2}$ years and histology showed small fistulous tracts lined by ciliated epithelium of primitive respiratory tract character, confirming that the lesions were remnants of branchial clefts. $\mathrm{He}$ has also had delayed language development, requiring speech therapy. His hearing is normal.

The couple's second child was born with a mild degree of microphthalmia and iris colobomata. No haemangiomas were evident, but on examination at 2 weeks of age, a cystic lesion had become apparent under the right eyelid which continued to grow. By 9 months of age, this haemangiomatous cyst had begun to displace the lid downwards and the eyeball upwards (fig 3). The anterior two thirds of the cyst was excised at 1 year of age. This child is now 2 years of age and there has been no apparent regrowth. She appears to have some useful vision in her left eye. In addition to her ophthalmological signs, she has a prominent fat

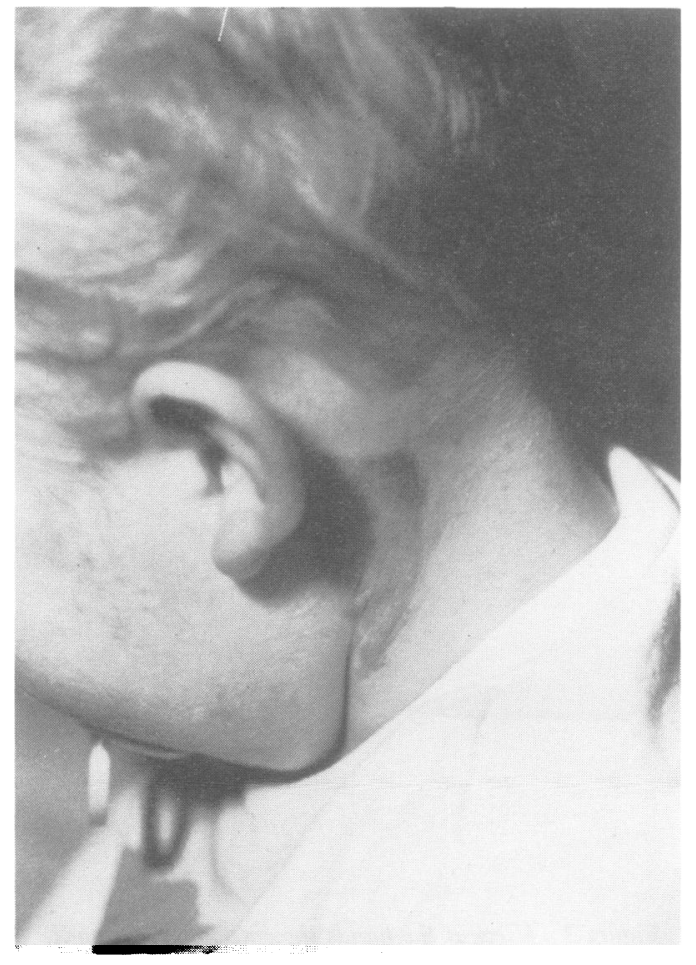

Figure 1 Haemangiomatous lesion of the neck in the older sib. 


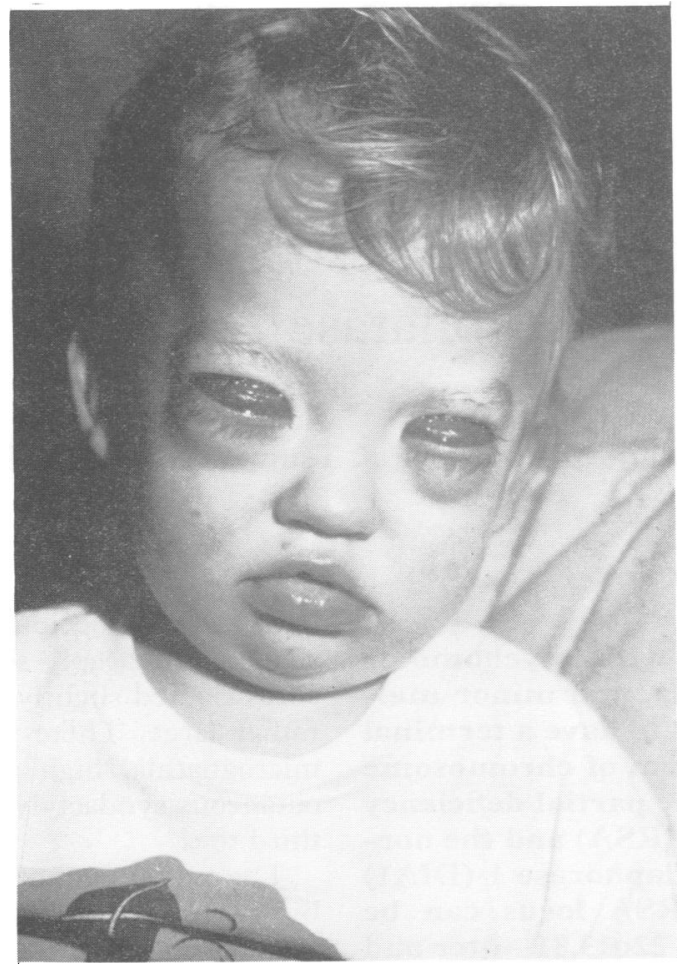

Figure 2 Facial appearance of the older sib before surgery showing haemangiomatous orbital cysts and pseudocleft of the lip.

pad on her chin and a short philtrum with prominent vertical ridges. Her eyes are normal. There are tiny non-haemangiomatous branchial sinuses which occasionally weep with crusting.

Neither parent has any physical signs of the condition and both have had a full ophthalmological assessment.

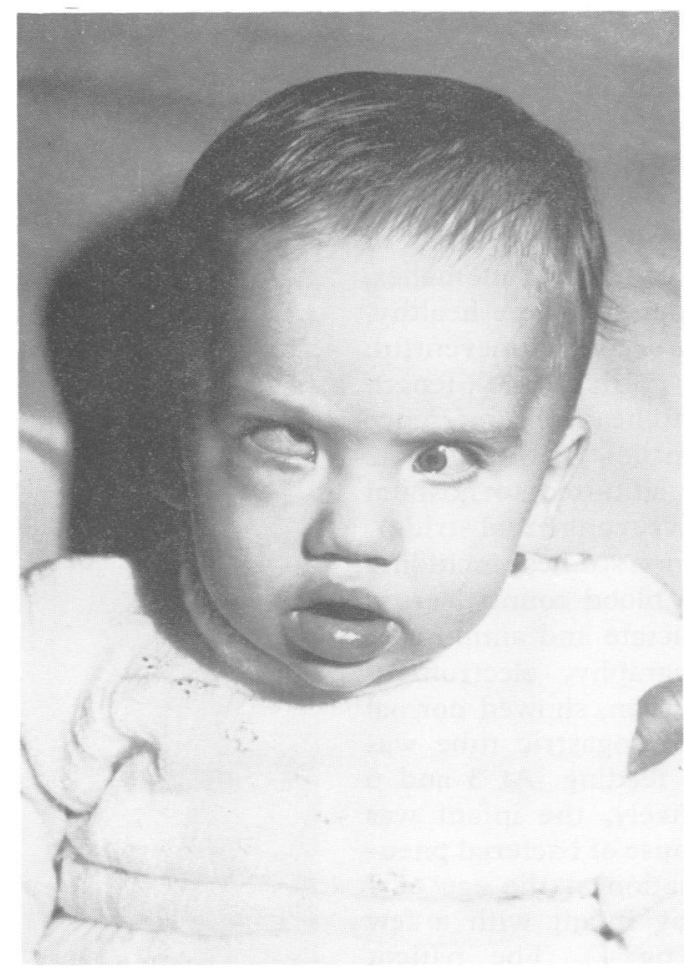

Figure 3 Facial appearance of the younger sib before surgery showing upward displacement of right eyeball by haemangiomatous orbital cyst.

\section{Discussion}

The presence of orbital lesions, haemangiomatous branchial cysts, and the characteristic facies with pseudocleft lip in this family fits with the previous reports of the branchiooculo-facial syndrome. The unusual feature in our family is the presence of haemangiomatous orbital cysts. Such lesions are rare. Orbital cysts have been reported in a few syndromes, including Dellemann syndrome, Goldenhaar syndrome, NAME syndrome, and Schopf syndrome although, to the best of our knowledge, haemangiomatous cysts have only been reported in Dellemann syndrome. The other features in our family support the diagnosis of the branchio-oculo-facial syndrome.

The branchio-oculo-facial syndrome has been regarded as an autosomal dominant trait on the basis of two reports of vertical transmission. Fujimoto et $a^{2}$ reported six cases in three families, in each case an affected parent having an affected child, and in one of the families there was father to son transmission. The clinical features in these families consisted of an abnormal upper lip (resembling a poorly repaired cleft), malformed nose with broad ridge and flattened tip, lacrimal duct obstruction, malformed ears, and branchial cleft sinuses or linear skin lesions behind the ears (haemangiomatous in two cases). Microphthalmia and iris coloboma were also noted in three of the six cases. Premature greying of the hair was noted in all three of the affected adults. These cases are convincingly similar to the original case described by Hall et $a l^{1}$ and to our own cases. Lee $e t a l^{3}$ reported an 8 year old boy who had non-haemangiomatous branchial cleft sinuses, obstruction of the nasolacrimal duct, strabismus, and unusual facies with short philtrum but no pseudocleft. His mother had had branchial cleft sinuses, bilateral nasolacrimal duct obstruction, and strabismus. She had premature greying of the hair. Both had been of low birth weight. It is possible that this family have the same condition.

All of the other reported cases have been sporadic and hence probably represent new dominant mutations. In our family the parents are clinically normal and so this could represent an autosomal recessive form of the condition, perhaps indicated by the severity of the ocular problems and the presence of the orbital cysts which have not been present in the other reports. The alternative explanation is that one or other parent is a germline mosaic for this dominant mutation. This disorder could then be added to the growing list of conditions for which germline mosaicism is being implicated.

We would like to thank Professor Bob Gorlin and Dr Dian Donnai for help in making the diagnosis in this family.

1 Hall BD, de Lorimier A, Foster LH. A new syndrome of haemangiomatous branchial clefts, lip pseudoclefts, and abnormal facial appearance. Am $\mathcal{F}$ Med Genet 1984;14:135-8.

2 Fujimoto A, Lipson M, Lacro RV, et al. New autosoma dominant branchio-oculo-facial syndrome. $A m \mathcal{F} \mathrm{Med}$ Genet 1987; 27:943-51.

3 Lee WK, Root AW, Fenske N. Bilateral branchial cleft sinuses associated with intrauterine and postnatal growth retardation, premature aging and unusual facial appearance: a new syndrome with dominant transmission. $A m \mathcal{F}$ Med Genet 1982;11:345-52. 